\title{
Numerical exploration of diffusion-controlled solid-state reactions in cubic particles
}

\author{
Phillip Gwo-Yan Huang ${ }^{a}$, Chung-Hsin Lu ${ }^{b}$, Tony Wen-Hann Sheu ${ }^{a}$ ** \\ a Department of Engineering Science and Ocean Engineering, National Taiwan University, Taipei, Taiwan, ROC \\ ${ }^{\mathrm{b}}$ Department of Chemical Engineering, National Taiwan University, Taipei, Taiwan, ROC
}

Received 30 April 2003; accepted 30 April 2003

\begin{abstract}
The characteristics of diffusion-controlled solid-state reactions in cubic particles are explored in this study. In order to capture the three-dimensional reaction front, the diffusion equation, subject to an interfacial chemical reaction in cubic particles, is solved in a transient form using the finite volume method. How the unreacted core is shrunk with time is clearly revealed. In addition, the concentration distributions of reactants and products are numerically predicted. A comparison of reacted zones advancing along different directions from the particle surface to the center is also made. The simulated kinetic curves at different conversion constants are shown to be useful to evaluate the conversion kinetics of diffusion-controlled solid-state reactions in cubic particles. (C) 2003 Elsevier B.V. All rights reserved.
\end{abstract}

Keywords: Cubic particles; Diffusion-controlled; Solid-state reactions; Finite volume method; Conversion constants; Conversion kinetics

\section{Introduction}

Analysis of solid-state reactions in particles has been the subject of an extensive investigation. Various kinetic models have been proposed for linking the degree of conversion $(\alpha)$ with time $(t)$ in order to determine the kinetics of solid-state reactions. These models can be classified into three groups, namely, the diffusioncontrolled reaction models [1-4], the phase-boundary controlled reaction models [5], and the nucleationcontrolled reaction models [6-8]. In these models, the working equations for relating $\alpha$ with $t$ were derived in a system full of geometrically simple particles. For a particle of circular disk shape, the reaction from its flat surfaces can be analyzed by the one-dimensional model [4]. In the literature, there also exist several multidimensional models for analyzing reaction processes in cylinders $[4,5]$ and in spheres $[1-3,5]$. The simulated results from these mathematical models help to estimate the reacted fraction. However, the assumptions made on

\footnotetext{
* Corresponding author. Tel.: +886-2-2362-5470x246; fax: +886-22392-9885.

E-mail address: twhsheu@ntu.edu.tw (T.W.-H. Sheu).
}

the above mentioned particles limit the range of applications. To the best of authors' knowledge, solidstate reactions in cubic particles have not been investigated. Furthermore, the three-dimensional interaction phenomena in particles of cubic shape are too complex to make legitimate approximations in the derivation of these mathematical models.

In our previous study, diffusion-controlled solid-state reactions were simulated in spherical particles [9] using a fully conservative finite-volume model [10]. The diffusion equation, subject to an interfacial chemical reaction, was solved to obtain the conversion kinetics. In this study we consider a commonly encountered solidstate reaction kinetics in cubic particles. Since this analysis is not amenable to analytic solution, a threedimensional numerical study is, thus, inevitable for an in-depth exploration of reaction kinetics in cubic particles. Meanwhile, diffusion of reactants in each reacted zone is considered to predict the concentrations of reactants and products. Our goal is to estimate the time needed to complete the conversion (or the complete conversion time $\left(t_{c}\right)$ ).

In this study the three-dimensional reacted zone that gradually advances towards the center of the investigated cubic particle is simulated. The concentrations of 
reactants and products in the direction from particle surface towards the center are also calculated. The resulting concentration gradient for the investigated reactant in the reacted layer can provide a good understanding of the dilution process. Furthermore, we define a conversion constant $K$ as the ratio of the reactant diffusivity to the square of particle edge length. The kinetic curves $(\alpha$ vs. $t)$ at different values of $K$ are calculated in order to extend our knowledge about the influence of $K$ on the conversion kinetics of diffusioncontrolled solid-state reactions in cubic particles.

\section{Diffusion-controlled solid-state reactions}

\subsection{Conversion kinetics}

As schematic in Fig. 1(a and b), isothermal solid-state reaction in a cubic particle of length $l_{o}$ is considered. The particle under investigation initially contains the reactant $B$, whose outer surface is thoroughly surrounded by the diffusing reactant $A$. At $t>0, A$ immediately reacts with $B$ according to the reaction $A+B \rightarrow A B$, thus producing a product layer $A B$ which covers the unreacted core of $B$. The reaction is followed by a diffusion process of $A$ into the product layer, and is assumed to occur instantaneously as $A$ reaches the interface of reacted and unreacted zones. Under these conditions, the product layer forms and propagates towards the center of the particle. In the conversion process, the diffusion velocity of $A$ is smaller than the chemical reaction velocity. Therefore, the diffusion process essentially controls the kinetics of solid-state reactions.

The solid-state reaction kinetics to be investigated can be well represented by the relationship between the degree of conversion $\alpha$ and time $t$. In this study $\alpha$ is defined as

$\alpha=\frac{A B_{r}}{A B_{o}}$

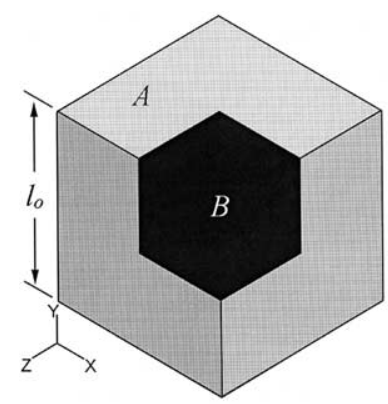

(a)

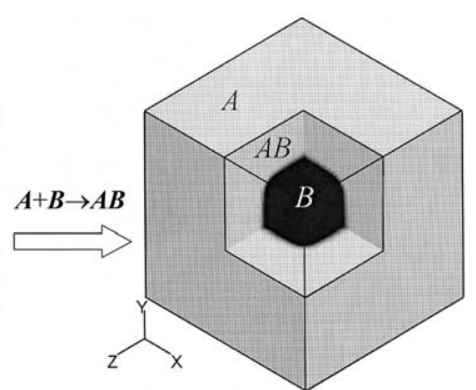

(b)
Fig. 1. The diffusion-controlled solid-state reaction $A+B \rightarrow A B$ in a cubic particle (a) at an initial state, and (b) when a reacted layer $A B$ is produced. where $A B_{r}$ is the reacted mole (or weight) of the product at time $t$. As for $A B_{o}$, it represents the total mole (or weight) of the product when the conversion is completed.

\subsection{Physical model}

In the course of diffusion-controlled solid-state reaction, the concentration of reactant $A$ in the product layer $A B$ varies with time and space. The unsteady transfer of species can be governed by the timedependent diffusion equation. In a notation-free coordinate system, the diffusion equation for $\mathrm{A}$ is expressed as:

$\frac{\partial\left(\rho C_{A}\right)}{\partial t}-\nabla\left(\rho D \nabla C_{A}\right)=0$

where $\rho$ is the particle density, $C_{A}$ the concentration of $A$, and $D$ the diffusivity of $A$ in the product layer. In the constant coefficient case, equation (2) can be further simplified as

$\frac{\partial C_{A}}{\partial t}-D \nabla^{2} C_{A}=0$

Equation (3) needs to be supplemented with a set of appropriate initial and boundary conditions. As depicted in Fig. 1(a and b), the particle initially contains a uniformly distributed reactant $B$, implying that $C_{A}=$ $C_{A B}=0$ inside the particle. The particle surface is always surrounded by $A$. A fixed concentration for reactant $A$ is, therefore, prescribed on the particle outer surface. In this study we consider the reaction $A+B \rightarrow$ $A B$. On the particle surface, $C_{A}$ is specified with the value of $C_{o}$. As for the initial value of $C_{B}$, it is taken as $C_{B}=C_{o}$. Therefore, the maximum concentration for the product $A B$ is $C_{A B}-C_{o}$.

The diffusion-controlled solid-state reaction is assumed to start immediately as $A$ diffuses into $A B$ and reaches the interface of $A B$ and unreacted $B$. According to the stoichiometric coefficients of reactants and product, the concentrations $C_{A}$ and $C_{B}$ for reactants and the concentration $C_{A B}$ for the product at the interface can be determined from

$C_{A}=\max \left(0, C_{A}^{*}-C_{B}^{*}\right)$

$C_{B}=\max \left(0, C_{B}^{*}-C_{A}^{*}\right)$

$C_{A B}=C_{A B}^{*}+\min \left(C_{A}^{*}, C_{B}^{*}\right)$

In the above, $C_{A}^{*}, C_{B}^{*}$ and $C_{A B}^{*}$ are the concentrations for $A, B$, and $A B$ at the reaction interface prior to the initiation of chemical reactions.

\subsection{Conversion constant}

In solid-state reactions, particles with the same conversion constant $K$ will result in the same conversion 
kinetics. The factor that characterizes the conversion kinetics is, thus, $K[1-8]$. In our previous study of solidstate reactions in spherical particles at isothermal conditions [9], $K$ is known to relate to the ratio of reactant diffusivity $(D)$ to the square of particle radius $\left(r_{o}^{2}\right)$. Here, we also define $K$ for cubic particles as

$K=\frac{D}{l_{o}^{2}}$

Therefore, $K$ represents the ratio of the diffusivity to the square of edge length $\left(l_{o}^{2}\right)$ of a cubic particle. In this paper, calculations are carried out at a variety of $K$ to predict the kinetic curves for diffusion-controlled solidstate reactions in cubic particles.

\section{Numerical model}

Equation (3) is integrated to obtain a discrete finitevolume equation that relates the solution, obtained at the center of the control volume, with its neighbors [10]. All spatial derivative terms are discretized using the second-order central scheme. In the present method, a fully implicit backward time stepping scheme is used to approximate the temporal derivative term [11].

Owing to the geometrical symmetry, only $1 / 8$ of the cubic particle, with $\mathrm{X}, \mathrm{Y}$, and $\mathrm{Z}$ being the planesymmetry schematic: in Fig. 2(a), needs to be analyzed. The physical domain is divided into hexahedral computational grids [12], as illustrated in Fig. 2(b). Calculations with increasing grid nodes are performed to obtain the grid-independent solution.

All computations are performed on a personal computer using some existing computer aided engineering softwares. A computer aided design (CAD) tool, namely, CATIA [13] is employed to create the cubic particle geometry. The initial conditions, boundary conditions, chemical reaction conditions and species properties are provided as the input data. The hexahedral grids are generated using a grid generator ICEMHEXA [14]. Species concentration and degree of conversion are calculated using the finite volume code CFX-

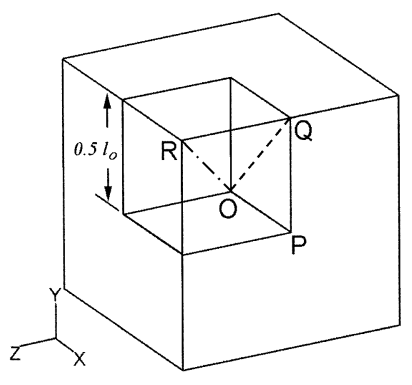

(a)

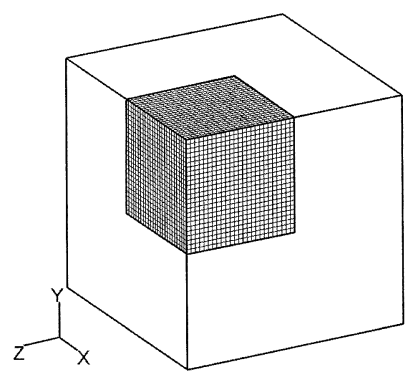

(b)
Fig. 2. (a) Schematic of the computational domain; (b) the distributed hexahedral grids.
4 [15]. Finally, the time-evolving product layer in the direction towards the particle center is revealed by virtue of the post-processor FIELDVIEW [16].

\section{Results and discussion}

\subsection{Grid-independent test}

For the investigated diffusion-controlled solid-state reaction in cubic particles, no exact solution is available for the validation of analysis code. Therefore, a number of calculations with increasing grid nodes should be performed to determine the grid-independent solution. In this study the complete conversion time $t_{c}$ at $K=5 \times$ $10^{-6} \mathrm{~s}^{-1}$ is used as the test problem. Fig. 3 illustrates the effect of grids on $t_{c}$. Clearly, $t_{c}$ obtained at different grids slightly vary with $5.20 \mathrm{~h}$. A typical analysis conducted in this paper involves 15625 grids, which result in $t_{c}=5.20 \mathrm{~h}$ at $K=5 \times 10^{-6} \mathrm{~s}^{-1}$.

\subsection{Simulations of the solid-state reaction}

In Fig. 4(a-c), we reveal the shrinkage of unreacted core at $1.0,2.0$, and $3.0 \mathrm{~h}$ for the case with $K=5 \times 10^{-6}$ $\mathrm{s}^{-1}$. At $1.0 \mathrm{~h}, A$ is seen to diffuse into the particle and react with reactant $B$, thus producing a product layer $A B$. The unreacted core of $B$, whose surface represents the front of $A B$, is plotted with $A B$ being hidden. Clearly seen from Fig. 4(a) is that both edge and corner of the unreacted core $B$ are sharply smeared. Following the formation of $A B$, reactant $A$ keeps diffusing through the product layer $A B$ and reaching the surface of the unreacted $B$. The solid-state reaction occurs instantaneously at the interface. Then, the unreacted core is seen to continuously shrink, as shown in Fig. 4(b and c). Figs. 5 and 6 present the advancement of the reacted layer from two different cross sectional planes.

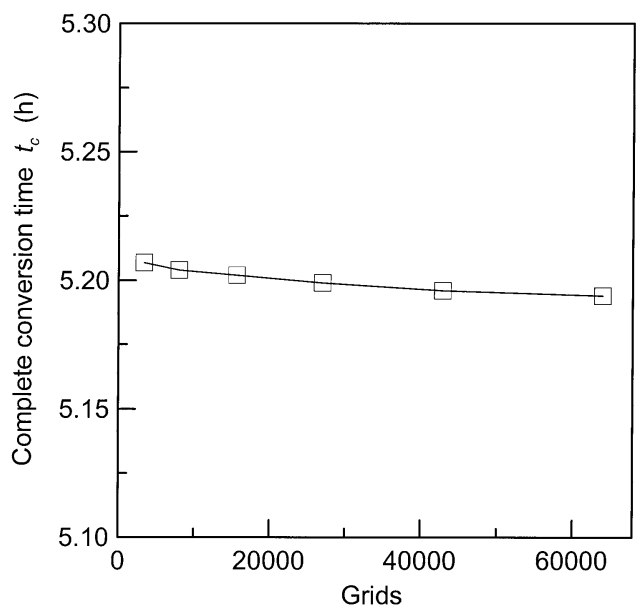

Fig. 3. An illustration of the effect of grids on the simulated complete conversion time $t_{c}$. 


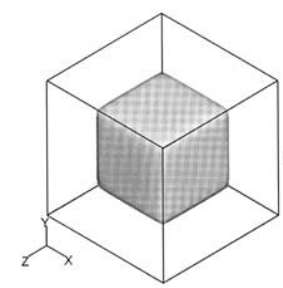

(a) $1.0 \mathrm{~h}$

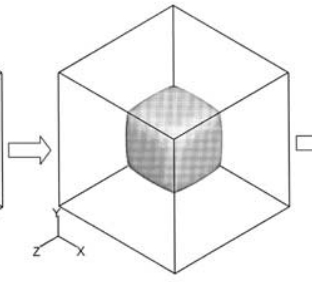

(b) $2.0 \mathrm{~h}$

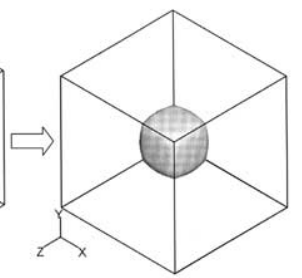

(c) $3.0 \mathrm{~h}$
Fig. 4. The time-evolving shrinkage of the unreacted core $B$ at times (a) $1.0 \mathrm{~h}$, (b) $2.0 \mathrm{~h}$, (c) $3.0 \mathrm{~h}$.

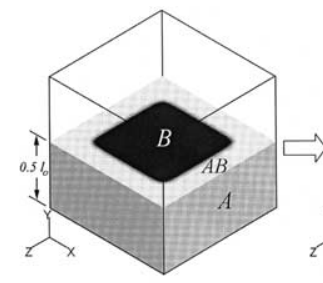

(a) $1.0 \mathrm{~h}$

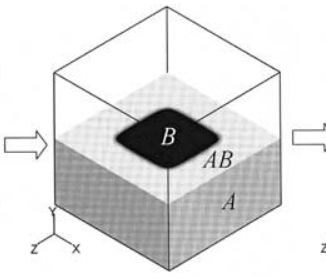

(b) $2.0 \mathrm{~h}$

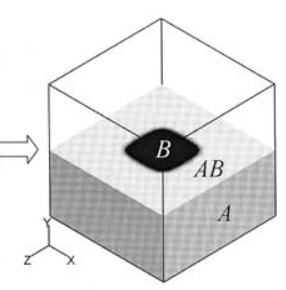

(c) $3.0 \mathrm{~h}$
Fig. 5. The time-evolving distributions of reacted layer $A B$ at times (a) $1.0 \mathrm{~h}$, (b) $2.0 \mathrm{~h}$, (c) $3.0 \mathrm{~h}$ on the $\mathrm{Y}=0.5 l_{o}$ plane.

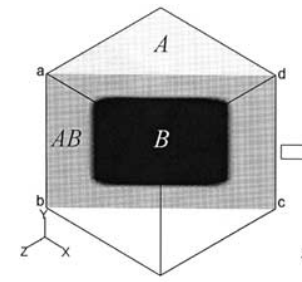

(a) $1.0 \mathrm{~h}$

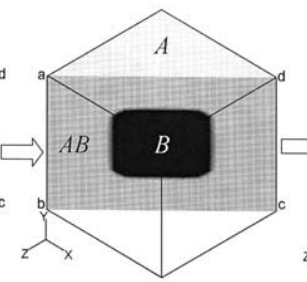

(b) $2.0 \mathrm{~h}$

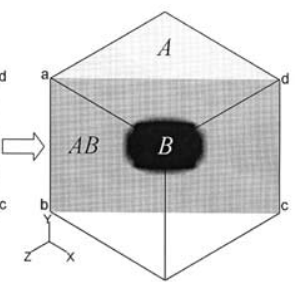

(c) $3.0 \mathrm{~h}$
Fig. 6. The time-evolving distributions of reacted layer $A B$ at times (a) $1.0 \mathrm{~h}$, (b) $2.0 \mathrm{~h}$, (c) $3.0 \mathrm{~h}$ on the $\mathrm{a}-\mathrm{b}-\mathrm{c}-\mathrm{d}$ diagonal plane.

For a cubic particle with uniform initial and boundary conditions, the shape of the unreacted core is seen to gradually develop into a spherical shape.

The reason why the edge and corner of the unreacted core are smeared with time can be explained by the simulated distribution of $A$ in the particle interior. Fig. 7 illustrates the distribution of $A$ at different periods of time on the surface of an inner cubic region of edge length $0.8 l_{o}$. Clearly, the normalized concentrations $C_{A}$ l $C_{o}$ on the edge and corner are greater than that on the face. The reason is that the edge receives $A$ from the two

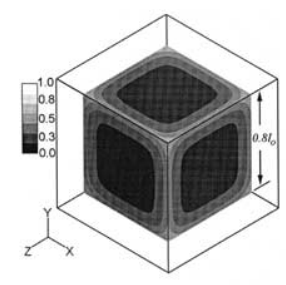

(a) $1.0 \mathrm{~h}$

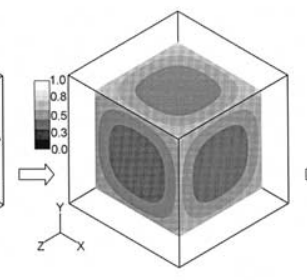

(b) $2.0 \mathrm{~h}$

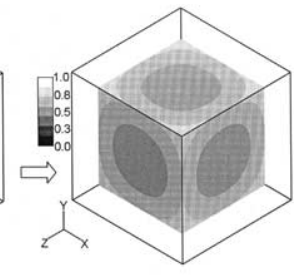

(c) $3.0 \mathrm{~h}$
Fig. 7. The normalized concentration distributions $\left(C_{A} / C_{o}\right)$ for reactant $A$ on the surface of an inner cubic region of edge length 0.8 $l_{o}$ at times (a) $1.0 \mathrm{~h}$, (b) $2.0 \mathrm{~h}$, (c) $3.0 \mathrm{~h}$. side faces, and the corner receives $A$ from its three neighboring faces. Therefore, a larger concentration of $A$ will be seen on the edge and corner. This will increase the consumption of $B$. The edge and corner are, therefore, sharply smeared.

Fig. $8(\mathrm{a}-\mathrm{c})$ present the normalized concentrations $\left(C_{i} / C_{o}, i=A, B\right.$, or $\left.A B\right)$ for reactants $A, B$ and product $A B$ against the normalized distance $\left(l / l_{o}\right)$ along the $\overline{O P}$ line, shown in Fig. 2(a), measured from the particle face to the center at 1.0, 2.0, and $3.0 \mathrm{~h}$, respectively, at $K=$ $5 \times 10^{-6} \mathrm{~s}^{-1}$. It is seen that $A$ in the product layer has an apparent concentration gradient. It implies that there is a diffusion of $A$ in the product layer due to this concentration gradient.

Fig. 9(a-c) depict the normalized concentrations $\left(C_{i} /\right.$ $C_{o}, i=A, B$ or $A B$ ) for $A, B$ and $A B$ against the normalized distance $\left(l / l_{o}\right)$ along the $\overline{O Q}$ line, shown in Fig. 2(a), measured from the particle edge to the center at $1.0,2.0$ and $3.0 \mathrm{~h}$, respectively, at $K=5 \times 10^{-6} \mathrm{~s}^{-1}$. Along $\overline{O Q}$, a concentration gradient for $A$ is seen in the product layer. The concentration of $A$ near the edge has a higher value, because this region can receive $A$ from two neighboring faces. The variation of $C_{A}$ along $\overline{O Q}$ is higher than that along $\overline{O P}$ (Fig. 8).

Fig. 10 (a-c) show the normalized concentrations $\left(C_{i} /\right.$ $C_{o}, i=A, B$ or $A B$ ) for $A, B$ and $A B$ against the normalized distance $\left(l / l_{o}\right)$ along $\overline{O R}$ line, shown in Fig.

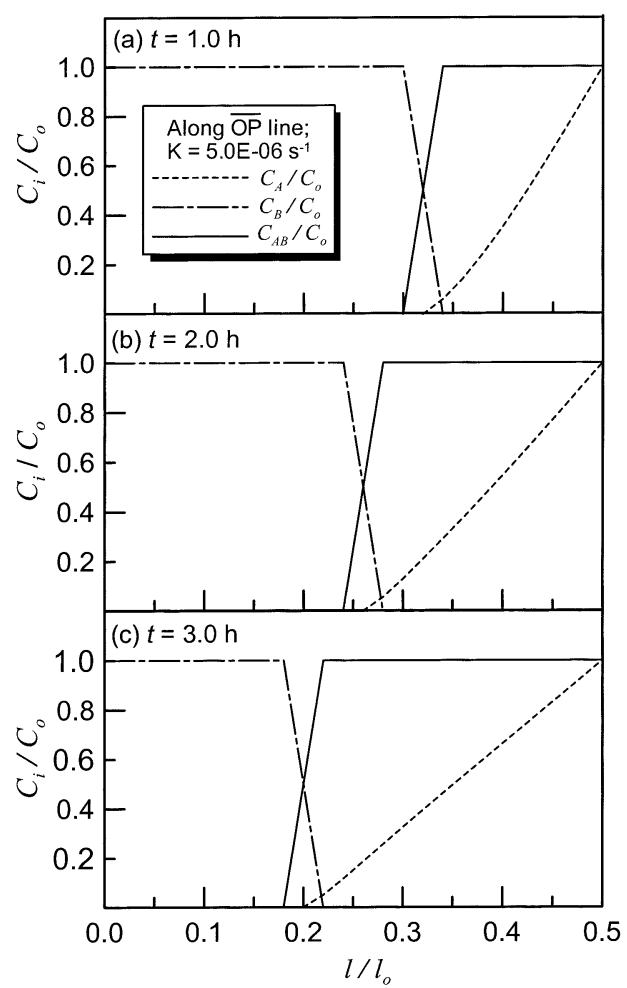

Fig. 8. The normalized concentration distributions $\left(C_{i} / C_{o}\right)$ for reactants $A, B$ and product $A B$, which are plotted against the normalized distance $\left(l / l_{o}\right)$ to the particle center along $\overline{O P}$ at times (a) $1.0 \mathrm{~h}$, (b) 2.0 h, (c) $3.0 \mathrm{~h}$. 


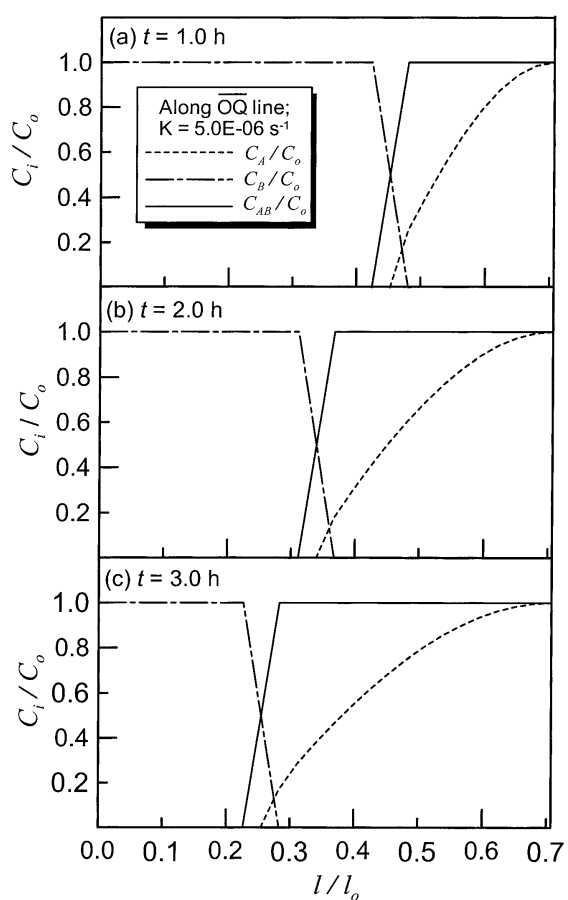

Fig. 9. The normalized concentration distributions $\left(C_{i} / C_{o}\right)$ for reactants $A, B$ and product $A B$, which are plotted against the normalized distance $\left(l / l_{o}\right)$ to the particle center along $\overline{O Q}$ at times (a) $1.0 \mathrm{~h}$, (b) 2.0 h, (c) $3.0 \mathrm{~h}$.

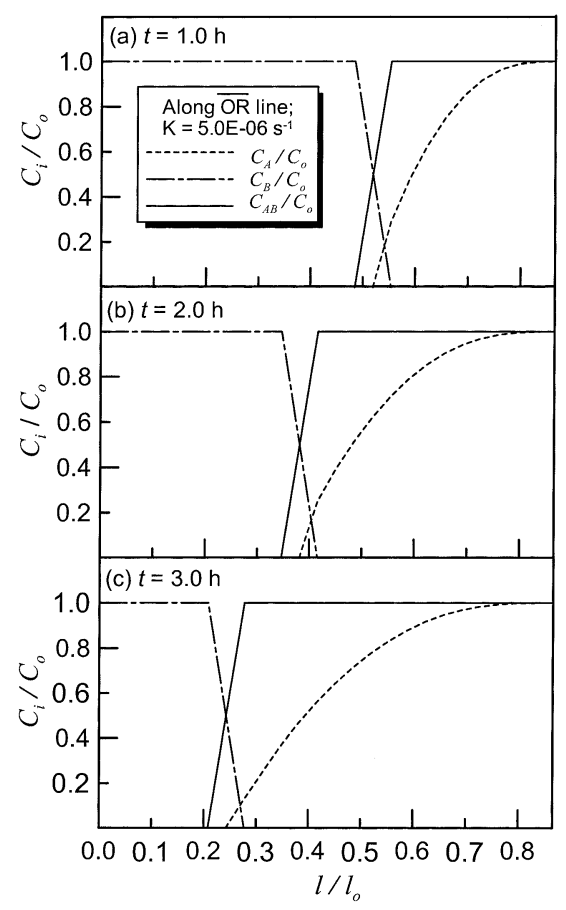

Fig. 10. The normalized concentration distribution $\left(C_{i} / C_{o}\right)$ for reactants $A, B$ and product $A B$, which are plotted against the normalized distance $\left(l / l_{o}\right)$ to the particle center along $\overline{O R}$ at times (a) $1.0 \mathrm{~h}$, (b) $2.0 \mathrm{~h}$, (c) $3.0 \mathrm{~h}$.

2(a), measured from the particle corner to the center at $1.0,2.0$ and $3.0 \mathrm{~h}$, respectively, at $K=5 \times 10^{-6} \mathrm{~s}^{-1}$. The concentration gradient for $\mathrm{A}$ along $\overline{O R}$ is also seen in the product layer. The concentration of $A$ around the corner has the highest value, because it receives $A$ from three neighboring faces. Therefore, the variation of $C_{A}$ along $\overline{O R}$ is higher than those along lines $\overline{O P}$ (Fig. 8) and $\overline{O Q}$ (Fig. 9).

Besides, it can be observed from Figs. 8-10 that the concentration gradient of $A$ becomes smaller with time. It means that it is more difficult for $A$ to diffuse into the increasingly thicker product layer. The concentration of $B$ remains unchanged in the unreacted zone and $C_{B}=0$ in the product layer. The distribution of $C_{A B}$ has the same trend as $C_{B}$, since the production of $A B$ is equal to the consumption of $B$. At the interface between the product layer $A B$ and unreacted $B$, there is a discontinuous distribution since reaction takes place there. In addition, we compare the normalized thickness $\left(l / l_{o}\right)$ of the reacted layers along lines $\overline{O P}$ (Fig. 8), $\overline{O Q}$ (Fig. 9) and $\overline{O R}$ (Fig. 10) at the same time. The thickness of the reacted layer along $\overline{O R}$ is found to have a larger value than that along $\overline{O Q}$ and $\overline{O P}$. This reveals that the conversion speed in the direction from the particle corner to the center has the highest value since the corner receives more reactant $A$ from its three neighboring faces.

Another comparison of the normalized thickness $\left(l / l_{o}\right)$ of the unreacted core along lines $\overline{O P}, \overline{O Q}$ and $\overline{O R}$ at different periods of time is shown in Fig. 11. As time marches, the difference in the thickness of unreacted core in three directions become smaller. This indicates that as the conversion proceeds, the original cubic core will be gradually evolved to show a spherical shape.

\subsection{Effect of conversion constant}

The kinetic curves obtained at different $K$ are presented in Fig. 12. All the simulated kinetic curves are shown to have a parabolic form. Initially, $\alpha$ is seen

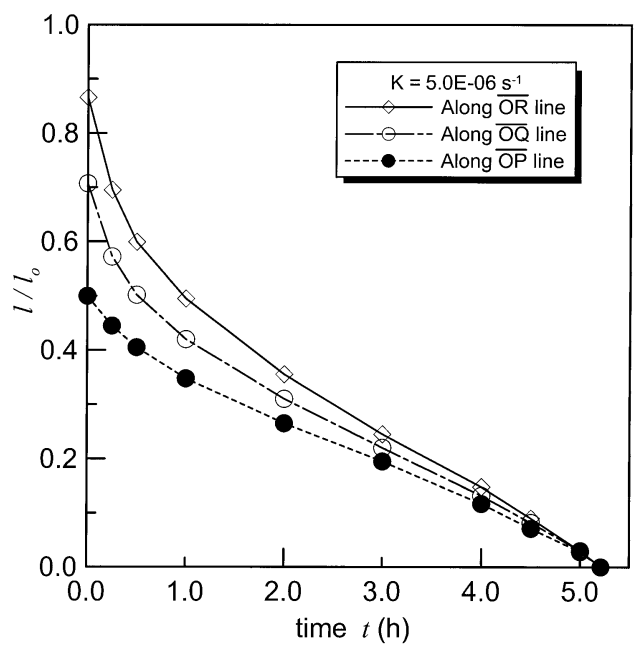

Fig. 11. A comparison of normalized thickness $\left(l / l_{o}\right)$ of the unreacted core along lines $\overline{O P}, \overline{O Q}$ and $\overline{O R}$ at several different periods of time. 


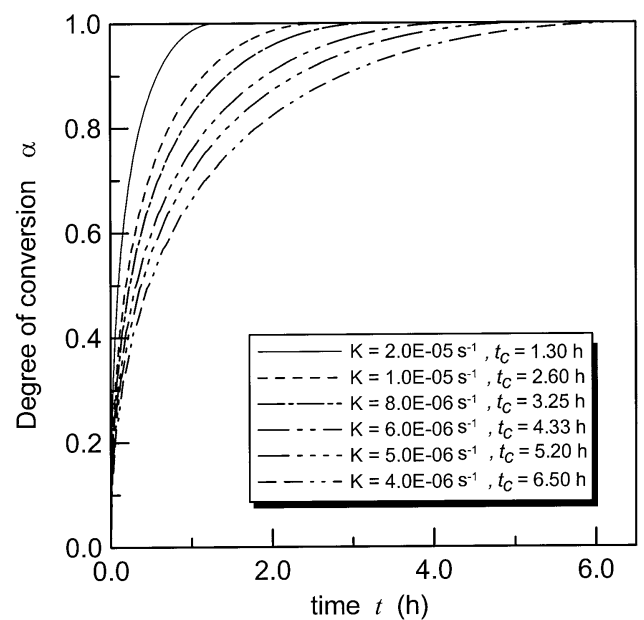

Fig. 12. The simulated kinetic curves (the degree of conversion $\alpha$ vs. time $t$ ) and the complete conversion times $\left(t_{c}\right)$ at different conversion constants $K$.

to increase considerably with $t$. This is followed by a much slower increase in $\alpha$ as the conversion proceeds. Because the concentration gradient of $A$ decreases when the product layer thickness increases. It will be more difficult for $A$ to diffuse into the thicker product layer. Fig. 12 also shows the effect of $K$ on the kinetics of conversion in cubic particles. It is obvious that $\alpha$ has a higher value for the case with a higher value of $K$. The ratio of two different $K$ is the inverse of the ratio of two simulated values of $t_{c}$ (complete conversion time). Furthermore, recall that the conversion constant $K$ defined in Eq. (7) represents the ratio of the reactant diffusivity to the square of the particle edge length. Cubic particles having the same value of $D / l_{o}^{2}$ will possess the same kinetics of conversion.

\section{Conclusions}

The diffusion-controlled solid-state reactions in cubic particles are numerically explored to reveal the shrinkage of unreacted core and the advancement of product layer. The conversion speed in the direction from the particle corner to the center is seen to be faster than those along the directions from the particle edge to the center and the particle face to the center. This difference will cause the unreacted core to change from the cubic shape to be spherical shape. The simulated concentrations for reactants and product in the conversion process enable us to investigate the unsteady diffusion behavior and explore the growth of product layer. The decreasing concentration gradient of the diffusing reactant in the product layer reveals the difficulty for the reactant to diffuse into the thicker product layer. The conversion constant $K$ defined as the ratio of the reactant diffusivity to the square of particle edge characterizes the conversion kinetics of diffusion-controlled solid-state reaction in cubic particles. The present study reveals that a larger $K$ will lead to a higher degree of conversion at the same time. The ratio of two different $K$ is the inverse of the ratio of two simulated values of the complete conversion time. For cubic particles with the same value of $K$ (or $D / l_{o}^{2}$ ), the same kinetics of conversion will be obtained.

\section{References}

[1] W. Jander, Z. Anorg. Allg. Chem. 163 (1927) 1.

[2] A.M. Ginstling, B.I. Brounshtein, J. Appl. Chem. USSR 23 (1950) 1327.

[3] R.E. Carter, J. Chem. Phys. 34 (1961) 2010.

[4] J.B. Holt, I.B. Cutler, M.E. Wadsworth, J. Am. Ceram. Soc. 45 (1962) 133.

[5] C. Wagner, Trans. AIME 192 (1952) 214.

[6] M. Avrami, J. Chem. Phys. 7 (1939) 1103

[7] B.V. Erofe'ev, Compt. Rend. Acad. Sci. USSR 52 (1946) 511.

[8] W.A. Johnson, R.F. Mehl, Trans. AIME 135 (1939) 416.

[9] P.G.Y. Huang, C.H. Lu, T.W.H. Sheu, communicated, 2003.

[10] S.V. Patankar, Numerical Heat Transfer and Fluid Flow, Hemisphere, 1983.

[11] D.B. Spalding, Int. J. Num. Methods Eng. 4 (1972) 551.

[12] J.F. Thompson, Z.U. Warsi, C.W. Mastin, Numerical Grid Generation, 1985.

[13] IBM and Dassault Systems, CATIA V5, 2002.

[14] ICEM CFD Engineering, ICEM-HEXA V4.2, 2002.

[15] AEA Technology, CFX-4.4, 2002.

[16] Intelligent Light, Fieldview V8, 2002. 